\title{
Intermittent Time-Varying Formation Control for High-Order Networked Agents Subject to Discontinuous Communications
}

\author{
Lixin Wang, Zhe Luo $i$, Xiaoqiang Li, Xinsan Li, and Xiaogang Yang $(\mathbb{D}$ \\ High-Tech Institute of Xi'an, Xi'an 710025, China \\ Correspondence should be addressed to Xiaogang Yang; doctoryxg@163.com
}

Received 10 December 2020; Revised 1 February 2021; Accepted 23 February 2021; Published 3 March 2021

Academic Editor: Ning Cai

Copyright (C) 2021 Lixin Wang et al. This is an open access article distributed under the Creative Commons Attribution License, which permits unrestricted use, distribution, and reproduction in any medium, provided the original work is properly cited.

\begin{abstract}
This paper investigates the leaderless and leader-follower time-varying formation design and analysis problems for a group of networked agents subject to discontinuous communications. Firstly, a leaderless time-varying formation control protocol is proposed via the intermittent control strategy, where the control input of each agent is constructed by the distributed local state information and formation instructions in the communication time unit, but it is zero in the noncommunication time unit. Then, an explicit formulation of the formation center function is determined to describe the formation movement trajectory of the whole networked agents. Leaderless time-varying formation design and analysis with discontinuous communications are given in the form of linear matrix inequalities. Moreover, the main results of the leaderless cases are extended to the leader-follower cases. Finally, two numerical examples are provided to illustrate the theoretical results of leaderless and leader-follower cases, respectively.
\end{abstract}

\section{Introduction}

Distributed cooperative control has received more attention from scholars in the last two decades, which can be applied in many circumstances, including flocking [1], consensus [2-6], formation control [7-10], distributed computation [11-13], and multisource data analysis $[14,15]$. Distributed formation control indicates that a group of networked intelligent agents form the desired geometrical shape via the distributed control protocol, which is constructed by the local information among neighboring agents. It was shown in [16] that the consensus-based formation control is distributed and can be achieved by utilizing the consensus algorithms and tools. Recently, the consensus theory developed fast, and many interesting research results emerged, as shown in [17-22]. As a result, the distributed formation control has aroused many researches, which can be divided into time-invariant formation and time-varying formation according to the time-dependent characteristics of the formation shape.

For the time-invariant formation, the relative position among agents remains unchanged after forming the formation structure, which means that the geometrical shape of the whole networked agents is time-invariant. From the graph theory perspective, the time-invariant formation control conditions were derived in the form of the Nyquist criterion in [23]. Jafarian et al. [24] investigated the timeinvariant formation keeping problems for nonholonomic wheeled robots, where the disturbance rejection is achieved. Finite-time time-invariant formation control was achieved in [25], where a class of nonlinear control protocol was utilized. For the time-varying formation, the formation shape can be time-varying, as shown in [26,27], which is more flexible than the time-invariant formation, and can be utilized in many practical applications. Dong et al. [28] provided a time-varying formation tracking scheme for second-order networked agents and applied it to the formation flying of a team of quadrotors. Wang et al. [29] proposed a robust time-varying formation control protocol with a distributed extend state observer, which can compensate the external disturbances actively. For a group of agents with multiple leaders, a leader-follower time-varying formation control method was shown in [30], where necessary and sufficient conditions with the formation tracking 
feasibility conditions were given. Generally speaking, the time derivative of the time-varying formation is not zero and cannot be ignored in formation design and analysis, so the time-varying cases are more challenging than the time-invariant ones.

Due to the temporary interrupt of communication links, the sensing device failures, and the silent period of communications, the networked agents may suffer discontinuous communications. On the one hand, the communication topologies may be switched since the links of the network are changed. Some interesting works regarding switching topologies can be found in [31, 32], where it was shown that the communication topologies switch at some moments by the switching signal. On the other hand, the discontinuous communications are intermittent in the sequence of the time units; that is, the communication time units and the noncommunication time units appear alternately. Wang et al. [33] investigated the limited-budget consensus problems with intermittent interactions, where the consensus protocol was codesigned by the limited budget and the performance index and can guarantee the weighting optimization between the consensus performance and the energy consumption. Sun and Wang [34] proposed a new sampling-based time unit method to solve the consensus problems for nonlinear networked agents with intermittent interactions. The time-invariant formation control with intermittent interactions was studied in [35]. However, to the best of our knowledge, the time-varying formation control problems for networked agents with discontinuous communication in terms of both the switching topologies and intermittent interactions are still open.

In this paper, we investigate the leaderless and leaderfollower time-varying formation design and analysis for high-order networked homogeneous agents with discontinuous communications caused by switching topologies and intermittent interactions. Firstly, a new time-varying formation control protocol is proposed via the intermittent control strategy, which only adopts the local intermittent information and formation instructions among neighboring agent. Secondly, by the nonsingular transformation and the orthonormal transformation, the closed-loop dynamics of the whole network for both leaderless cases and leaderfollower cases are decomposed into two subdynamics, which, respectively, describe the formation movement trajectory of the networked agents as a whole and the relative movement among agents. Thirdly, leaderless and leaderfollower time-varying formation design and analysis criteria are derived under the condition of discontinuous communications, where the convergency of the Lyapunov function is analyzed in the communication time units and noncommunication time units, and it can be guaranteed by satisfying the formation feasibility condition, the discontinuous communication condition, and the linear matrix inequality condition, simultaneously.

Compared with the related work regarding the timevarying formation control, the contribution of this paper is twofold. Firstly, different from the works in [26-30], this paper considers the discontinuous communications of both switching topologies and intermittent communications. In this case, the right-hand side of the closed-loop system is piecewise continuous. To solve this problem, a new intermittent time-varying formation control method is proposed. However, the analysis and design method in [26-30] cannot be adopted in this paper. Secondly, this paper determines the formation movement trajectory of the networked agents for both leaderless and leader-follower cases. For the leaderless case, the formation movement trajectory is determined by the formation center function, and it is determined by the zero-input response of the leader in the leader-follower case. Besides, it is shown that the intermittent communication and the switching topology do not affect the formation movement trajectory. In contrast, the authors in [26-30] did not determine the formation movement trajectory in the situation of the discontinuous communications.

The main body of this paper is arranged as follows. The model of discontinuous communications and the dynamics of the agents are established in Section 2. Leaderless timevarying formation design and analysis criteria with discontinuous communications are given in Section 3, where the explicit formulation of the formation center function is also determined. Section 4 extends the main results of the leaderless time-varying formation design and analysis to the leader-follower cases. Two numerical simulation examples are provided in Section 5, and Section 6 concludes the whole paper.

Throughout this paper, $\mathfrak{R}^{n \times d}$ stands for the $n \times d$-dimensional real matrix space. $\aleph$ is the set of natural numbers. $N$ is utilized to denote the number of the agents, and $1_{N}$ represents the $N$-dimensional column vector with all components 1 . The number, vector, and matrix of zero value are collectively called as $0 . Q^{T}=Q>0$ means that matrix $Q$ is symmetric and positive definite.

\section{Problem Formulation and Preliminaries}

2.1. Communication Constraint Modeling. In this paper, we consider the discontinuous communication among agents. On the one hand, the agent cannot communicate with each other in some noncommunication time units. On the other hand, the communication topologies of the networks are switched in some communication time units. To show the abovementioned discontinuous communication type from the time-domain perspective, it is supposed for $\forall s \in \mathcal{N}$ that there exists a nonoverlapping time unit sequence $\left[T_{s}, T_{s+1}\right)=\left[T_{s}, \widetilde{T}_{s}\right) \cup\left[\widetilde{T}_{s}, T_{s+1}\right)$, where $T_{s}=T_{s}^{1}<T_{s}^{2}<\cdots<$ $T_{s}^{r_{s}}=\widetilde{T}_{s}<\widetilde{T}_{s}+\varepsilon_{s}=T_{s+1}$ with $r_{s}$ and $\varepsilon_{s}$ being positive integers. Notice that $\left[T_{s}, \widetilde{T}_{s}\right)$ and $\left[\widetilde{T}_{s}, T_{s+1}\right)$ represent the communication time unit and the noncommunication time unit, respectively. $T_{s}^{1}, T_{s}^{2}, \ldots, T_{s}^{r_{s}} \ldots$ denotes the switching time over which the communication topologies are switched. Without loss of generality, the initial time is assumed to be $T_{0}=0$. The length of time unit $\left[T_{s}, T_{s+1}\right)$ satisfies that $0<T_{\min }^{*} \leq T_{s}^{*}=T_{s+1}-T_{s} \leq T_{\max }^{*}$. It should be

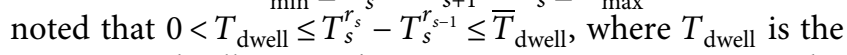
minimum dwell time. The noncommunication rate is defined as $\sigma_{s}=\left(T_{s+1}-\widetilde{T}_{s}\right) /\left(T_{s+1}-T_{s}\right)$, where $0<\sigma_{s} \leq \sigma_{\max }<1$ and $\sigma_{\max }$ is called the maximum noncommunication rate. Note that the discontinuous communication is aperiodic 
since the length of each time unit $\left[T_{s}, \widetilde{T}_{s}\right)(s \in \aleph)$ can be unequal.

The switching topologies are modeled as $\mathbb{G}=\left\{G^{1}, G^{2}, \ldots, G^{k}\right\} \quad$ with the switching signal $\Phi(t):[0,+\infty) \longrightarrow\{1,2, \ldots, k\}$, where $G$ is the digraph. For each digraph $G$, the vertex set is denoted by $\mathscr{V}=\left\{v_{1}, v_{2}, \ldots, v_{H}\right\}$, and the edge set is represented by $\mathscr{E}=$ $\left\{\left(v_{l}, v_{m}\right): v_{l}, v_{m} \in \mathscr{V}\right\}$ with the edge weight $b_{l m}^{\Phi(t)}$. Note that if there exists an edge $\left(v_{l}, v_{m}\right), v_{l}, v_{m} \in \mathscr{V}$, from the vertex $v_{m}$ to $v_{l}$, then the edge weight $b_{l m}^{\Phi(t)}>0$. Otherwise, $b_{l m}^{\bowtie(t)}=0$. The neighboring set of the vertex $v_{l}$ is defined as $\mathcal{N}_{l}^{\Phi(t)}=\left\{v_{m} \in \mathscr{V}:\left(v_{m}, v_{l}\right) \in \mathscr{E}\right\}, \quad$ and $\quad L^{\Phi(t)}=\left[l_{l m}^{\Phi(t)}\right]_{H \times H}$ stands for the Laplacian matrix of the topologies with $l_{l l}^{\Phi(t)}=$ $\sum_{m \in \mathcal{N}_{l}^{\oplus(t)}} b_{l m}^{\Phi(t)}$ and $l_{l m}^{\Phi(t)}=-b_{l m}^{\Phi(t)}(l \neq m)$. More details about the graph theory can be found in [36]. It should be pointed out that the switching topology does not affect the requirement of the noncommunication rate. In this paper, both leaderless and leader-follower communication topologies are considered, which satisfy the following assumption.

Assumption 1. It is assumed that the leaderless communication topology is represented by connected undirected graph, and the leader-follower communication topology is denoted by digraph containing a spanning tree with the leader locating at the root of the spanning tree.

Lemma 1. For the connected undirected graph, the Laplacian matrix $L$ is symmetric and positive semidefinite, and zero is the simple eigenvalue of $L$.

2.2. Network Dynamics Modeling. The dynamics of the networked agents with leaderless structures are described as follows:

$$
\dot{x}_{l}(t)=A x_{l}(t)+B u_{l}(t),
$$

where $l=1,2, \ldots, N, A \in \mathfrak{R}^{n \times n}, B \in \mathfrak{R}^{n \times d}, x_{l}(t)$ are the states of agent $l$, and $u_{l}(t)$ is the control protocol.

Definition 1. The expected formation shape of the network is described by a vector-valued function $z(t)=\left[z_{1}^{T}(t)\right.$, $\left.z_{2}^{T}(t), \ldots, z_{N}^{T}(t)\right]^{T}$, where $z_{l}(t) \quad(l=1,2, \ldots, N)$ is the piecewise continuous differentiable and is called the formation instruction.

According to the formation instruction, we propose a time-varying formation control protocol via the intermittent control strategy as follows:

$$
u_{l}(t)= \begin{cases}K \sum_{m \in \mathcal{N}_{l}^{(0(t)}} b_{l m}^{\Phi(t)}\left(x_{m}(t)-z_{m}(t)-x_{l}(t)+z_{l}(t)\right), & t \in\left[T_{s}, \widetilde{T}_{s}\right), \\ 0, & t \in\left[\widetilde{T}_{s}, T_{s+1}\right),\end{cases}
$$

where $l=1,2, \ldots, N, K \in \mathfrak{R}^{d \times n}$ is the gain matrix. Then, the definitions of the leaderless time-varying formation design and analysis are given as follows.

Definition 2. (leaderless time-varying formation analysis). For any given gain matrix $K \in \mathfrak{R}^{d \times n}$ and bounded initial states $x_{l}(0)-z_{l}(0), l=1,2, \ldots, N$, if there exists a function $h(t) \in \mathfrak{R}^{n}$ such that $\lim _{t \rightarrow+\infty}\left(x_{l}(t)-z_{l}(t)-h(t)\right)=0$, $l=1,2, \ldots, N$, then it is said that network (1) with protocol (2) reaches leaderless time-varying formation, where $h(t)$ is said to be the formation center function.

Definition 3. (leaderless time-varying formation design). If there exists a gain matrix $K$ such that network (1) with protocol (2) reaches leaderless time-varying formation, then it is said to be leaderless time-varying formation reachable.

In this paper, we mainly focus on designing the gain matrix $K$ such that network (1) subject to discontinuous communications reaches leaderless time-varying formation with protocol (2). Then, the main results on the leaderless time-varying formation are extended to the leader-follower cases.
Remark 1. It should be noticed that protocol (2) is constructed via the intermittent control strategy; that is, the control input is intermittent over the nonoverlapping time unit sequence $\left[T_{s}, T_{s+1}\right), \forall s \in \aleph$. In the communication time unit, the control input is established according to the local state information among neighboring agents and the formation instructions. However, in the noncommunication time unit, the control input is set to be zero since it is missing. This kind of intermittent control strategy will lead to the piecewise continuous right-hand side of the closed-loop networks, which is challenging to be dealt with in the stability analysis of the networked agents.

Remark 2. Note that, for the consensus, it requires that all the agents reach an agreement of states, where the formation structure is not needed. For the time-invariant formation, the formation instruction is invariable, whose time derivate is zero; i.e., $\dot{z}_{l} \equiv 0 \quad(l=1,2, \ldots, N)$. Compared with the consensus and the time-invariant formation, the main difficulty in designing the time-varying formation protocol is that the time derivate of the formation instruction $z_{l}(t)(l=$ $1,2, \ldots, N)$ affects the analysis of the convergency of the 
formation. In this case, the formation feasibility condition is introduced to overcome the challenging problems in designing the gain matrix of the time-varying formation protocol.

\section{Leaderless Time-Varying Formation Design and Analysis}

In this section, we give leaderless time-varying formation design and analysis criteria under the condition of discontinuous communications, and then, we determine an explicit formulation of the formation center function.

For $l=1,2, \ldots, N$, let $\varphi_{l}(t)=x_{l}(t)-z_{l}(t)$, substituting (2) into (1) gives that

$$
\dot{\varphi}_{l}(t)= \begin{cases}A\left(\varphi_{l}(t)+z_{l}(t)\right)+B K \sum_{m \in \mathcal{N}_{l}^{\infty(t)}} b_{l m}^{\Phi(t)}\left(\varphi_{m}(t)-\varphi_{l}(t)\right)-\dot{z}_{i}(t), & t \in\left[T_{s}, \widetilde{T}_{s}\right), \\ A\left(\varphi_{l}(t)+z_{l}(t)\right)-\dot{z}_{i}(t), & t \in\left[\widetilde{T}_{s}, T_{s+1}\right) .\end{cases}
$$

Denote $\varphi(t)=\left[\varphi_{1}^{T}(t), \varphi_{2}^{T}(t), \ldots, \varphi_{N}^{T}(t)\right]^{T}$, then we can rewrite equation (3) as

$$
\dot{\varphi}(t)= \begin{cases}\left(I_{N} \otimes A\right)(\varphi(t)+z(t))-\left(L^{\oplus(t)} \otimes B K\right) \varphi(t)-\left(I_{N} \otimes I_{n}\right) \dot{z}(t), & t \in\left[T_{s}, \widetilde{T}_{s}\right), \\ \left(I_{N} \otimes A\right)(\varphi(t)+z(t))-\left(I_{N} \otimes I_{n}\right) \dot{z}(t), & t \in\left[\widetilde{T}_{s}, T_{s+1}\right) .\end{cases}
$$

Since Assumption 1 holds, we can find from Lemma 1 that there exists an orthonormal matrix $W^{\varpi(t)}=\left[1_{N} / \sqrt{N}, \widetilde{W}^{\varpi(t)}\right]$ such that

$$
\left(W^{\varpi(t)}\right)^{T} L^{\varpi(t)} W^{\varpi(t)}=\operatorname{diag}\left\{\lambda_{1}^{\varpi(t)}, \lambda_{2}^{\varpi(t)}, \ldots, \lambda_{N}^{\varpi(t)}\right\},
$$

where $0=\lambda_{1}^{\oplus(t)}<\underline{\lambda} \leq \lambda_{2}^{\oplus(t)} \leq \lambda_{3}^{\oplus(t)} \leq \cdots \leq \lambda_{N}^{\oplus(t)} \leq \bar{\lambda}$ are the eigenvalues of the Laplacian matrix. Denote $\Omega^{\Phi(t)}=\operatorname{diag}\left\{\lambda_{2}^{\Phi(t)}, \lambda_{3}^{\Phi(t)}, \ldots, \lambda_{N}^{\Phi^{(t)}}\right\}$ and $v(t)=\left(\left(W^{\Phi(t)}\right)^{T}\right.$ $\left.\otimes I_{n}\right) x(t)=\left[v_{1}^{T}(t), \kappa^{T}(t)\right]^{T}$, where $\kappa^{T}(t)=\left[v_{2}^{T}(t), v_{3}^{T}(t)\right.$, $\left.\ldots, v_{N}^{T}(t)\right]^{T}$, and then, we can transform network (4) into the following form:

$$
\begin{aligned}
& \dot{v}_{1}(t)=A v_{1}(t)+\left(\frac{1_{H}^{T}}{\sqrt{H}} \otimes A\right) z(t)-\left(\frac{1_{H}^{T}}{\sqrt{H}} \otimes I_{n}\right) \dot{z}(t), \quad t \in\left[T_{s}, T_{s+1}\right), \\
& \dot{\kappa}(t)= \begin{cases}\left(I_{H-1} \otimes A-\Omega^{\Theta(t)} \otimes B K\right) \kappa(t)+\left(\left(\widetilde{W}^{\Theta(t)}\right)^{T} \otimes A\right) z(t)-\left(\left(\widetilde{W}^{\Theta(t)}\right)^{T} \otimes I_{n}\right) \dot{z}(t), & t \in\left[T_{s}, \widetilde{T}_{s}\right), \\
\left(I_{H-1} \otimes A\right) \kappa(t)+\left(\left(\widetilde{W}^{\Theta(t)}\right)^{T} \otimes A\right) z(t)-\left(\left(\widetilde{W}^{\Theta(t)}\right)^{T} \otimes I_{n}\right) \dot{z}(t), & t \in\left[\widetilde{T}_{s}, T_{s+1}\right) .\end{cases}
\end{aligned}
$$

From the above orthonormal transformation, network (4) is decomposed into two subnetworks; i.e., subnetworks (6) and (7). In the following, we will provide a theorem to describe the explicit formulation of the formation center function according to the dynamics of subnetwork (6).

Theorem 1. If network (1) with protocol (2) reaches the timevarying formation $z(t)$, then the formation center function satisfies that

$$
\lim _{t \longrightarrow+\infty}\left(h(t)-h_{c}(t)+h_{z}(t)\right)=0,
$$

where

$$
\begin{aligned}
& h_{c}(t)=e^{A t}\left(\frac{1_{N}^{T}}{N} \otimes I_{n}\right) x(0), \\
& h_{z}(t)=\left(\frac{1_{N}^{T}}{N} \otimes I_{n}\right) z(t) .
\end{aligned}
$$


Proof. Define the following functions:

$$
\begin{aligned}
& \Gamma_{c}(t) \triangleq W^{\oplus(t)} e_{1} \otimes v_{1}(t)=\frac{1}{\sqrt{H}} 1_{H} \otimes v_{1}(t), \\
& \Gamma_{z}(t) \triangleq \sum_{l=2}^{H} W^{\varpi(t)} e_{l} \otimes v_{l}(t),
\end{aligned}
$$

where $e_{l}(l \in\{1,2, \ldots, N\})$ is a $N$-dimensional unit column vector, whose $l$ th element is 1 . According to equation (10), we have

$$
\Gamma_{c}(t)=\left(W^{\varrho(t)} \otimes I_{n}\right)\left[v_{1}^{T}(t), 0\right]^{T} .
$$

Since

$$
\sum_{l=2}^{N} W^{\mathrm{\omega}(t)} e_{l} \otimes v_{l}(t)=\left[0, \kappa^{T}(t)\right]^{T},
$$

we can derive that

$$
\Gamma_{z}(t)=\left(W^{\oplus(t)} \otimes I_{n}\right)\left[0, \kappa^{T}(t)\right]^{T} .
$$

Because $W^{\oplus(t)} \otimes I_{n}$ is a nonsingular matrix, we can see from the above analysis that $\Gamma_{c}(t)$ and $\Gamma_{z}(t)$ are linearly independent. Hence, we can use $v_{1}(t) / \sqrt{N}$ to determine the explicit formulation of the formation center function.

According to Definition 2 and equation (12), we have

$$
\lim _{t \longrightarrow+\infty}\left(x_{l}(t)-z_{l}(t)-\frac{1}{\sqrt{N}} v_{1}(t)\right)=0 \text {. }
$$

From equation (16), we have

$$
v_{1}(0)=\left(\left(\frac{1}{\sqrt{N}} 1_{N}^{T}\right) \otimes I_{n}\right) \varphi(0) .
$$

Then, we can derive that

$$
\begin{aligned}
& \int_{0}^{t} e^{A(t-\tau)}\left(\left(\frac{1}{\sqrt{N}} 1_{N}^{T}\right) \otimes I_{n}\right) \dot{z}(\tau) \mathrm{d} \tau \\
& =\left(\left(\frac{1}{\sqrt{N}} 1_{N}^{T}\right) \otimes I_{n}\right) z(t)-e^{A t}\left(\left(\frac{1}{\sqrt{N}} 1_{N}^{T}\right) \otimes I_{n}\right) z(0) \\
& \quad+\int_{0}^{t} e^{A(t-\tau)}\left(\left(\frac{1}{\sqrt{N}} 1_{N}^{T}\right) \otimes A\right) z(\tau) \mathrm{d} \tau .
\end{aligned}
$$

From equations (6), (16), and (17), we can obtain the conclusion of Theorem 1.

Remark 3. The explicit formulation of the formation center function in Theorem 1 describes the formation movement trajectory of the networked agents as a whole. Note that when the time-varying formation is reached, all the networked agents will keep a formation shape and move along with the trajectory determined by the formation center function, which contains two parts. The first part $h_{c}(t)$ is associated with the dynamics and the initial states of each agent, which describe the influence mechanism of the consensus states on the formation movement. The second part $h_{z}(t)$ is related to the timevarying formation instruction. From the formulation of $h(t)$, we can find that the discontinuous communication does not impact the formation movement trajectory of the whole networked agents.

According to the analysis from (10) to (14), we can conclude that network (1) reaches time-varying formation if and only if $\lim _{t \rightarrow+\infty} \kappa(t)=0$. Based on this fact, we give the time-varying formation design criterion in the following theorem.

Theorem 2. Network (1) is leaderless time-varying formation reachable by protocol (2) with $K=0.5 \underline{\lambda}^{-1} \vartheta B^{T} \widetilde{Q}^{-1}$ if the following conditions hold simultaneously:

(i) The formation feasibility condition $\dot{z}_{l}(t)=A z_{l}(t)$ $(l=1,2, \ldots, N)$ holds.

(ii) The discontinuous communication condition $\alpha(1-$ $\left.\sigma_{\max }\right)>\mu \sigma_{\max }$ is satisfied, where $\alpha$ and $\mu$ are positive constants given previously.

(iii) There exist $\vartheta>0$ and $\widetilde{Q}=\widetilde{Q}^{T}>0$ such that

$$
\begin{array}{r}
A \widetilde{Q}+\widetilde{Q} A^{T}-\mu \widetilde{Q}<0, \\
A \widetilde{Q}+\widetilde{Q} A^{T}+\alpha \widetilde{Q}-\vartheta B B^{T}<0 .
\end{array}
$$

Proof. Construct the Lyapunov function as follows:

$$
V(t)=\kappa^{T}(t)\left(I_{N-1} \otimes \widetilde{Q}^{-1}\right) \kappa(t)
$$

For $t \in\left[T_{s}, \widetilde{T}_{s}\right)$ with $\forall s \in \aleph$, we can obtain the time derivative of $V(t)$ according to the dynamics of subnetwork (7) that

$$
\begin{aligned}
\dot{V}(t)= & \kappa^{T}(t)\left(I_{N-1} \otimes\left(\widetilde{Q}^{-1} A+A^{T} \widetilde{Q}^{-1}\right)\right. \\
& \left.-\Omega^{\Phi(t)} \otimes\left(\widetilde{Q}^{-1} B K+K^{T} B^{T} \widetilde{Q}^{-1}\right)\right) \kappa(t) \\
& +2 \kappa^{T}(t)\left(\left(\widetilde{W}^{\Phi(t)}\right)^{T} \otimes \widetilde{Q}^{-1} A\right) z(t) \\
& -2 \kappa^{T}(t)\left(\left(\widetilde{W}^{\Phi(t)}\right)^{T} \otimes \widetilde{Q}^{-1}\right) \dot{z}(t) .
\end{aligned}
$$

Since the formation feasibility condition $\dot{z}_{l}(t)=A z_{l}(t)$ $(l=1,2, \ldots, N)$ holds, we can deduce that $\left(\left(\widetilde{W}^{\omega^{(t)}}\right)^{T} \otimes \widetilde{Q}^{-1}\right) \dot{z}(t)=\left(\left(\widetilde{W}^{\Phi(t)}\right)^{T} \otimes \widetilde{Q}^{-1} A\right) z(t)$. Then, we can see from (20) that

$$
\begin{aligned}
\dot{V}(t)= & \kappa^{T}(t)\left(I_{N-1} \otimes\left(\widetilde{Q}^{-1} A+A^{T} \widetilde{Q}^{-1}\right)\right. \\
& \left.-\Omega^{\Phi(t)} \otimes\left(\widetilde{Q}^{-1} B K+K^{T} B^{T} \widetilde{Q}^{-1}\right)\right) \kappa(t) .
\end{aligned}
$$

Substituting $K=0.5 \underline{\lambda} \vartheta B^{T} \widetilde{Q}^{-1}$ into equation (21) yields 


$$
\begin{aligned}
\dot{V}(t)+\alpha V(t)= & \sum_{l=2}^{N} \kappa_{l}^{T}(t)\left(\widetilde{Q}^{-1} A+A^{T} \widetilde{Q}^{-1}+\alpha \widetilde{Q}^{-1}\right. \\
& \left.-\lambda_{l}^{\Phi(t)} \underline{\lambda}^{-1} \vartheta \widetilde{Q}^{-1} B B^{T} \widetilde{Q}^{-1}\right) \kappa_{l}(t) .
\end{aligned}
$$

By pre- and postmultiplying $A \widetilde{Q}+\widetilde{Q} A^{T}+\alpha \widetilde{Q}-\vartheta B B^{T}<0$ with $\widetilde{Q}^{-1}$ and $-\lambda_{l}^{\oplus(t)} \underline{\lambda}^{-1} \leq 1(l=2,3, \ldots, N)$, we can obtain from (22) that

$$
\dot{V}(t)<-\alpha V(t) .
$$

For $t \in\left[\widetilde{T}_{s}, T_{s+1}\right)$ with $\forall s \in \aleph$, we can obtain the time derivative of $V(t)$ along the dynamics of subnetwork (7) that

$$
\dot{V}(t)=\kappa^{T}(t)\left(I_{N-1} \otimes\left(\widetilde{Q}^{-1} A+A^{T} \widetilde{Q}^{-1}\right)\right) \kappa(t) .
$$

Then, it follows that

$$
\dot{V}(t)-\mu V(t)=\sum_{l=2}^{N} \kappa_{l}^{T}(t)\left(\widetilde{Q}^{-1} A+A^{T} \widetilde{Q}^{-1}-\mu \widetilde{Q}^{-1}\right) \kappa_{l}(t) .
$$

According to $A \widetilde{Q}+\widetilde{Q} A^{T}-\mu \widetilde{Q}<0$, we have

$$
\dot{V}(t)<\mu V(t) .
$$

In the sequel, we discuss the convergency of $V(t)$ along the time unit sequence $\left[T_{s}, T_{s+1}\right), \forall s \in \aleph$. Firstly, for $t \in\left[T_{0}, T_{1}\right)$, we can show that

$$
\begin{aligned}
V\left(T_{1}\right) & <e^{\mu\left(T_{1}-\widetilde{T}_{0}\right)} V\left(\widetilde{T}_{0}\right)<e^{\mu\left(T_{1}-\widetilde{T}_{0}\right)} e^{-\alpha\left(\widetilde{T}_{0}-T_{0}\right)} V\left(T_{0}\right) \\
& =e^{-\beta_{0}} V(0),
\end{aligned}
$$

where $\beta_{0}=\left(\alpha-(\alpha+\mu) \sigma_{0}\right) T_{0}^{*}$. By the discontinuous communication condition $\alpha\left(1-\sigma_{\max }\right)>\mu \sigma_{\max }$, we can see that $\beta_{0}>0$. Then, we have for any positive integer $s$ that

$$
V\left(T_{s+1}\right)<V(0) e^{-\sum_{q=0}^{s} \beta_{q}},
$$

where $\beta_{q}=\left(\alpha-(\alpha+\mu) \sigma_{q}\right) T_{q}^{*}, q=1,2, \ldots, s$. For any $t>0$, we can see that an integer $i \geq 1$ exists such that $T_{i}<t \leq T_{i+1}$. Hence, we have

$$
\begin{gathered}
V(t) \leq e^{\mu T_{\max }^{*}} V\left(T_{i}\right) \leq e^{\mu T_{\max }^{*}} V(0) e^{-\sum_{j=0}^{i-1} \beta_{j}} \leq e^{\mu T_{\max }^{*}} \\
\cdot V(0) e^{-i\left(\alpha-(\alpha+\mu) \sigma_{\max }\right) T_{\min }^{*}} \\
\leq e^{\mu T_{\max }^{*}} V(0) e^{-\left(\left(\left(\alpha-(\alpha+\mu) \sigma_{\max }\right) T_{\min }^{*}\right) / T_{\max }^{*}\right) t} .
\end{gathered}
$$

According to equation (29), we can conclude that $\lim _{t \longrightarrow+\infty} \kappa(t)=0$, which means that network (1) reaches time-varying formation exponentially. The proof of Theorem 2 is finished.

The time-varying formation design criterion in Theorem 2 provides an approach to design the gain matrix of protocol (2). However, if the gain matrix is given previously, then it is interesting to check that the given gain matrix is feasible or not. Let $Q=\widetilde{Q}^{-1}$, then the following corollary gives the time-varying formation analysis criterion.

Corollary 1. For any given gain matrix $K$, network (1) with protocol (2) reaches leaderless time-varying formation if the following conditions hold simultaneously:

(i) The formation feasibility condition $\dot{z}_{l}(t)=A z_{l}(t)$ $(l=1,2, \ldots, N)$ holds.

(ii) The discontinuous communication condition $\alpha(1-$ $\left.\sigma_{\max }\right)>\mu \sigma_{\max }$ is satisfied, where $\alpha>0$ and $\mu>0$.

(iii) There exist $Q=Q^{T}>0$ such that

$$
\begin{array}{r}
Q A+A^{T} Q-\mu Q<0, \\
Q A+A^{T} Q+\alpha Q-\underline{\lambda}\left(Q B K+K^{T} B^{T} Q\right)<0, \\
Q A+A^{T} Q+\alpha Q-\bar{\lambda}\left(Q B K+K^{T} B^{T} Q\right)<0 .
\end{array}
$$

Remark 4. The formation feasibility condition in Theorem 2 and Corollary 1 indicates that not all the desired formation instructions can be reached by the networked agents. We can find intuitively that the formation structure to be formed is restrained by the dynamics of each agent. For example, due to the constraint of the maneuvering characteristics, a team of unmanned aerial vehicles cannot perform some specific actions and thus cannot form the corresponding formation shape. For the time-varying formation, some formation feasibility conditions similar to the condition $\dot{z}_{l}(t)=A z_{l}(t)$ $(l=1,2, \ldots, N)$ can be found in [28-30]. Note that if the derivative of the time-varying formation instruction is zero; i.e., $\dot{z}_{l}(t) \equiv 0,(l=1,2, \ldots, N)$, then the formation feasibility condition becomes $A z_{l}(t)=0$. In this case, the formation instruction is time-invariant.

Remark 5. Since the intermittent control strategy leads to the piecewise continuous right-hand side of the closed-loop systems, the stability property of the closed-loop systems should be analyzed in the communication time units and the noncommunication time units. It should be pointed out that the states of the closed-loop systems may be divergent in the noncommunication time units. To ensure the whole convergency of the Lyapunov function, the discontinuous communication condition $\alpha\left(1-\sigma_{\max }\right)>\mu \sigma_{\max }$ is provided, which can establish the relationship between the convergency factor $\alpha$ and the divergent factor $\mu$ via the maximum noncommunication rate $\sigma_{\max }$. As the result, the Lyapunov function $V(t)$ can be convergent with the rate faster than $\left(\alpha-(\alpha+\mu) \sigma_{\max }\right) T_{\min }^{*} / T_{\max }^{*}$ in virtue of inequality (29).

\section{Extensions to Leader-Follower Cases}

This section extends the main results of the leaderless timevarying formation design and analysis with discontinuous communications to the leader-follower cases.

The dynamics of networked agents with leader-follower structures are modeled as 


$$
\left\{\begin{array}{l}
\dot{x}_{N}(t)=A x_{N}(t), \\
\dot{x}_{l}(t)=A x_{l}(t)+B u_{l}(t),
\end{array}\right.
$$

where $l=1,2, \ldots, N-1$ are the labels of $N-1$ followers, and the leader is labeled by the subscript $N$.
Assumption 2. It is assumed that the communication topology among followers is represented by the connected undirected graph.

For leader-follower structures, we propose the following time-varying formation control protocol:

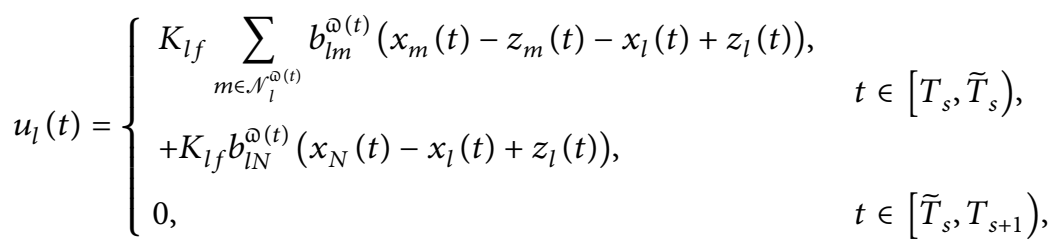

where $l=1,2, \ldots, N-1, K_{l f} \in \mathfrak{R}^{d \times n}$ is the gain matrix. Then, the definitions of the leader-follower time-varying formation design and analysis are given as follows.

Definition 4. (leader-follower time-varying formation anal$y$ sis). For any given gain matrix $K_{l f} \in R^{d \times n}$ and bounded initial states $x_{l}(0)-z_{l}(0), \quad l=1,2, \ldots, N-1, \quad$ if $\lim _{t \rightarrow+\infty}\left(x_{l}(t)-z_{l}(t)-x_{N}(t)\right)=0, \quad l=1,2, \ldots, N-1$, then it is said that network (1) with protocol (2) reaches leader-follower time-varying formation.

Definition 5. (leader-follower time-varying formation design). If there exists a gain matrix $K_{l f}$ such that network (1) with protocol (2) reaches leader-follower time-varying formation, then it is said to be leader-follower time-varying formation reachable.

Form (31) and (32), we can obtain that

$$
\dot{\varphi}(t)= \begin{cases}\left(I_{N} \otimes A\right)(\varphi(t)+z(t))-\left(L_{l f}^{\oplus(t)} \otimes B K_{l f}\right) \varphi(t)-\left(I_{N} \otimes I_{n}\right) \dot{z}(t), & t \in\left[T_{s}, \widetilde{T}_{s}\right), \\ \left(I_{N} \otimes A\right)(\varphi(t)+z(t))-\left(I_{N} \otimes I_{n}\right) \dot{z}(t), & t \in\left[\widetilde{T}_{s}, T_{s+1}\right),\end{cases}
$$

where $L_{l f}^{\Phi(t)}$ satisfies that

$$
\begin{aligned}
L_{l f}^{\Phi(t)} & =\left[\begin{array}{cc}
L_{f}^{\Phi(t)}+\Delta_{l}^{\Phi(t)} & -l_{l}^{\Phi(t)} \\
0 & 0
\end{array}\right], \\
\Delta_{l}^{\Phi(t)} & =\operatorname{diag}\left\{b_{1 N}^{\Phi(t)}, b_{2 N}^{\Phi(t)}, \ldots, b_{(N-1) N}^{\Phi(t)}\right\}, \\
l_{l}^{\Phi(t)} & =\left[b_{1 N}^{\Phi(t)}, b_{2 N}^{\Phi(t)}, \ldots, b_{(N-1) N}^{\Phi(t)}\right]^{T} .
\end{aligned}
$$
followers.

$L_{f}^{\complement(t)}$ is the Laplacian matrix of the topology among

Construct the following nonsingular matrix:

$$
Y^{\oplus(t)}=\left[\begin{array}{cc}
I_{N-1} & 1_{N-1} \\
0 & 1
\end{array}\right] .
$$
have

Let $\widetilde{\varphi}_{l}(t)=\varphi_{l}(t)-x_{N}(t) \quad(l=1,2, \ldots, N-1)$, then we

$$
\left(\left(Y^{\Phi(t)}\right)^{-1} \otimes I_{n}\right) \varphi(t)=\left[\widetilde{\varphi}_{2}^{T}(t), \ldots, \widetilde{\varphi}_{N}^{T}(t), x_{1}^{T}(t)\right]^{T} .
$$

Then, it follows from $\Delta_{l}^{\Phi(t)} 1_{N-1}=l_{l}^{\infty(t)}$ that

$$
\left(Y^{\Phi(t)}\right)^{-1} L^{\Phi(t)} Y^{\Phi(t)}=\left[\begin{array}{cc}
L_{f}^{\Phi(t)}+\Delta_{l}^{\Phi(t)} & 0 \\
0 & 0
\end{array}\right] .
$$

Since Assumptions 1 and 2 hold, we can find that $L_{f}^{\Phi(t)}+\Delta_{l}^{\Phi(t)}$ is positive definite and symmetric. Hence, there exists an orthonormal matrix $\widetilde{Y}^{\Phi(t)} \in \mathfrak{R}^{(N-1) \times(N-1)}$ such that

$$
\left(\widetilde{Y}^{\Phi(t)}\right)^{T}\left(L_{f}^{\Phi(t)}+\Delta_{l}^{\Phi(t)}\right) \widetilde{Y}^{\Phi(t)}=\Omega_{f}^{\Phi(t)}=\operatorname{diag}\left\{\widetilde{\lambda}_{1}^{\Phi(t)}, \tilde{\lambda}_{2}^{\Phi(t)}, \ldots, \widetilde{\lambda}_{N-1}^{\Phi(t)}\right\},
$$

where $0<\widetilde{\lambda}_{1}^{\Phi(t)} \leq \widetilde{\lambda}_{2}^{\Phi(t)} \leq \cdots \leq \widetilde{\lambda}_{N-1}^{\Phi(t)}$ are the eigenvalues of $L_{f}^{\Phi(t)}$ Let $\quad \widetilde{\varphi}(t)=\left[\widetilde{\varphi}_{1}^{T}(t), \widetilde{\varphi}_{2}^{T}(t), \ldots, \widetilde{\varphi}_{N-1}^{T}(t)\right]^{T} \quad$ and $\left(\left(Y^{\dot{\Phi}(t)}\right)^{-1} \otimes I_{n}\right) \widetilde{\varphi}(t)=\eta(t)=\left[\eta_{1}^{T}(t), \eta_{2}^{T}(t), \ldots, \eta_{N-1}^{T}(t)\right]^{T}$, then network (33) is converted to the following two subnetworks: 


$$
\begin{aligned}
\dot{x}_{N}(t) & =A x_{N}(t), \\
\dot{\eta}(t) & = \begin{cases}\left(I_{N-1} \otimes A-\Omega_{f}^{\Phi(t)} \otimes B K_{l f}\right) \eta(t)+\left(\left(\widetilde{Y}^{\oplus(t)}\right)^{T} \otimes A\right) z(t)-\left(\left(\widetilde{Y}^{\oplus(t)}\right)^{T} \otimes I_{n}\right) \dot{z}(t), & t \in\left[T_{s}, \widetilde{T}_{s}\right), \\
\left(I_{N-1} \otimes A\right) \eta(t)+\left(\left(\widetilde{Y}^{\Theta(t)}\right)^{T} \otimes A\right) z(t)-\left(\left(\widetilde{Y}^{\oplus(t)}\right)^{T} \otimes I_{n}\right) \dot{z}(t), & t \in\left[\widetilde{T}_{s}, T_{s+1}\right) .\end{cases}
\end{aligned}
$$

Because $Y^{\oplus(t)}$ and $\tilde{Y}^{\oplus(t)}$ are nonsingular, we can find that network (31) reaches leader-follower time-varying formation if and only if $\lim _{t \longrightarrow+\infty} \eta(t)=0$. Let $\underline{\lambda}_{l f}=\min \left\{\tilde{\lambda}_{1}^{i}: \forall i \in\{1,2, \ldots, k\}\right\}$ and $\bar{\lambda}_{l f}=\max \left\{\tilde{\lambda}_{1}^{i}: \forall i \in\right.$ $\{1,2, \ldots, k\}\}$, then we give the following theorem to show the sufficient conditions of the leader-follower time-varying formation design.

Theorem 3. Network (31) is leader-follower time-varying formation reachable by protocol (32) with $K_{l f}=0.5 \underline{\lambda}_{l f}^{-1}$ $\xi B^{T} \widetilde{R}^{-1}$ if the following conditions hold simultaneously:

(i) The formation feasibility condition $\dot{z}_{l}(t)=A z_{l}(t)$ $(l=1,2, \ldots, N)$ holds.

(ii) The discontinuous communication condition $\alpha(1-$ $\left.\sigma_{\max }\right)>\mu \sigma_{\max }$ is satisfied, where $\alpha>0$ and $\mu>0$.

(iii) There exist $\xi>0$ and $\widetilde{R}=\widetilde{R}^{T}>0$ such that

$$
\begin{array}{r}
A \widetilde{R}+\widetilde{R} A^{T}-\mu \widetilde{R}<0, \\
A \widetilde{R}+\widetilde{R} A^{T}+\alpha \widetilde{R}-\xi B B^{T}<0 .
\end{array}
$$

Corollary 2. For any given gain matrix $K_{l f}$, network (31) with protocol (32) reaches leader-follower time-varying formation if the following conditions hold simultaneously:

(i) The formation feasibility condition $\dot{z}_{l}(t)=A z_{l}(t)$ $(l=1,2, \ldots, N)$ holds.

(ii) The discontinuous communication condition $\alpha(1-$ $\left.\sigma_{\max }\right)>\mu \sigma_{\max }$ is satisfied, where $\alpha>0$ and $\mu>0$.

(iii) There exists $R=R^{T}>0$ such that

$$
\begin{array}{r}
R A+A^{T} R-\mu R<0, \\
R A+A^{T} R+\alpha R-\underline{\lambda}_{l f}\left(R B K_{l f}+K_{l f}^{T} B^{T} R\right)<0, \\
R A+A^{T} R+\alpha R-\bar{\lambda}_{l f}\left(R B K_{l f}+K_{l f}^{T} B^{T} R\right)<0 .
\end{array}
$$

Remark 6. The leader-follower time-varying formation can be regarded as an extension of the leaderless time-varying formation since their main conclusions are similar. In this case, the control gains of these two cases can be designed via solving the linear matrix inequalities in the similar form. However, the topology structures of these two cases are different, which can be reflected on the difference of the eigenvalues of the Laplacian matrices. Moreover, the formation trajectories of the leaderless time-varying formation and the leader-follower time-varying formation are different. For the leaderless case, the formation trajectory is determined by the states of all agents and the formation instruction, which is described by the formation center function. For the leader-follower case, the formation trajectory is determined by the leader.

\section{Numerical Simulations}

In this section, we provide two numerical simulation examples to demonstrate the effectiveness of the proposed theorems regarding the leaderless and leader-following time-varying formation design and analysis with discontinuous communications.

Example 1. (leaderless topologies). Consider a group of networked agents labeled by 1-6, which are of third order as follows:

$$
\begin{aligned}
& A=\left[\begin{array}{ccc}
0 & 1 & 0 \\
0 & 0 & 1 \\
0.5 & -1 & 0.5
\end{array}\right], \\
& B=\left[\begin{array}{l}
1 \\
2 \\
2
\end{array}\right] .
\end{aligned}
$$

The initial states of the agents are set as

$$
\begin{aligned}
& x_{1}(0)=[3.5,-5.1,-2.8]^{T}, \\
& x_{2}(0)=[-2.3,-7.5,1.6]^{T}, \\
& x_{3}(0)=[2.8,-4.6,1.3]^{T}, \\
& x_{4}(0)=[2.7,-2.1,2.1]^{T}, \\
& x_{5}(0)=[3.7,-1.4,-5.9]^{T}, \\
& x_{6}(0)=[7.2,-2.9,4.3]^{T} .
\end{aligned}
$$

The switching topologies are given in Figure 1, where the dwell time is set as $0.3 \mathrm{~s}$.

The communication time unit and noncommunication time unit are set to be $t \in[s, s+0.8) s$ and $t \in[s+0.8, s+1) s$, respectively, which are period time units for better operability of the simulation. In this case, the maximum noncommunication rate $\sigma_{\max }=0.2$. Choose $\alpha=1.6$ and $\mu=6$, and then, we can find that the discontinuous communication condition $\alpha\left(1-\sigma_{\max }\right)>\mu \sigma_{\max }$ is satisfied. The timevarying formation instruction is chosen as follows: 

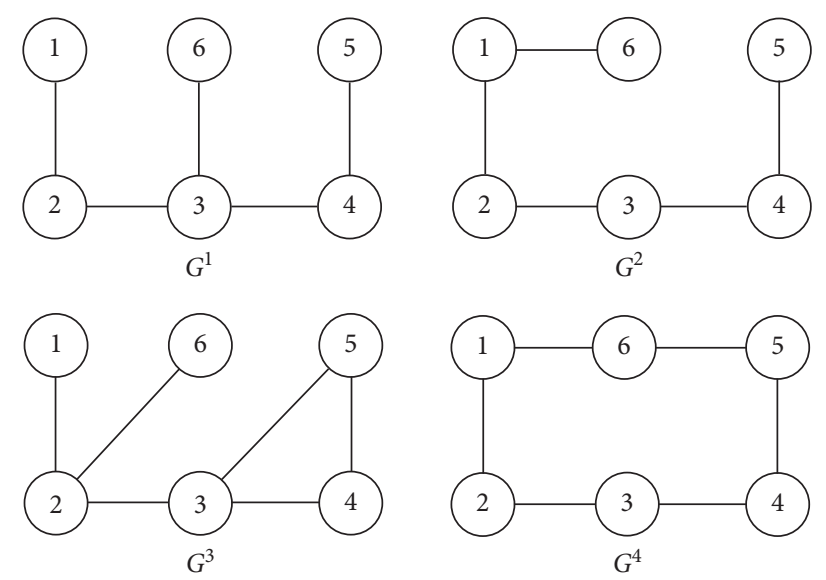

FIgURE 1: Leaderless switching topologies.
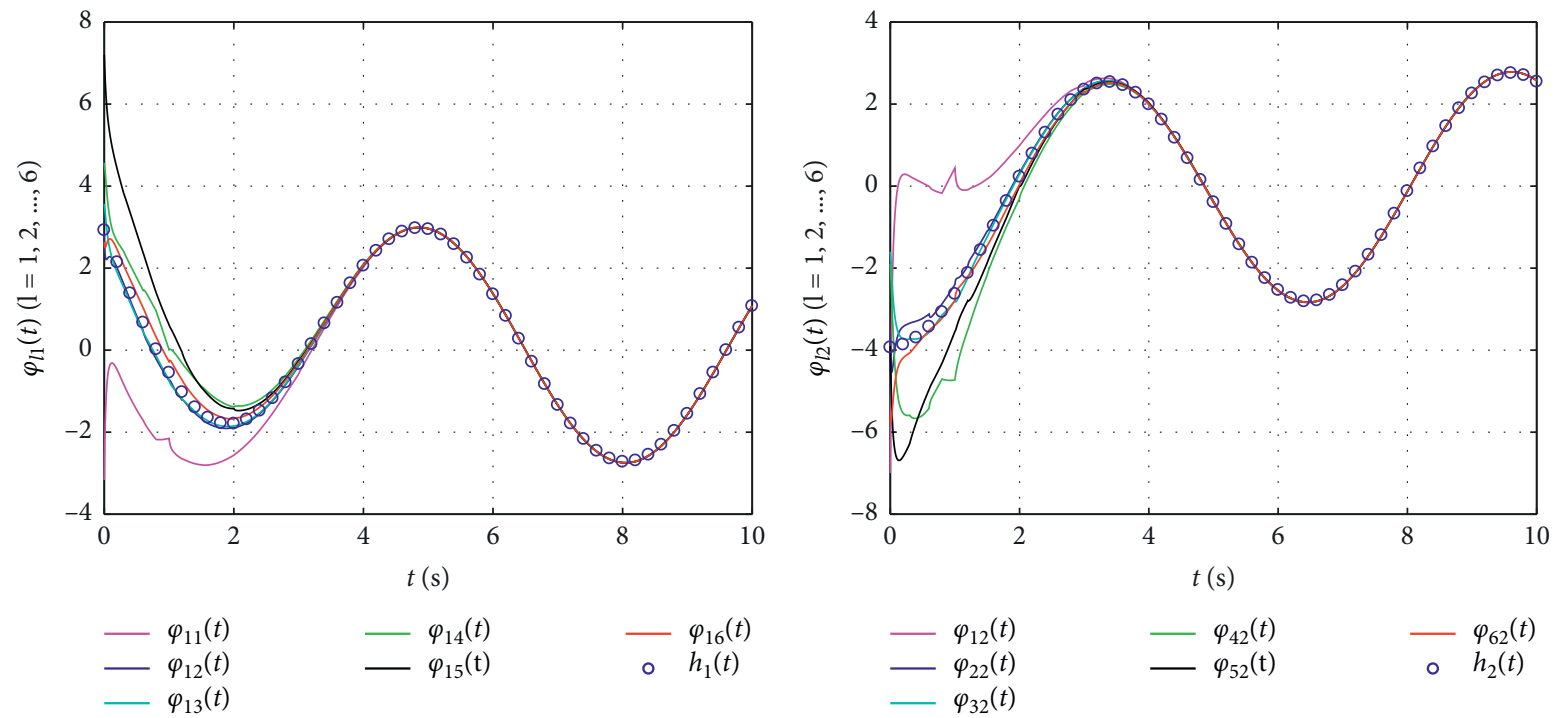

(a)

(b)

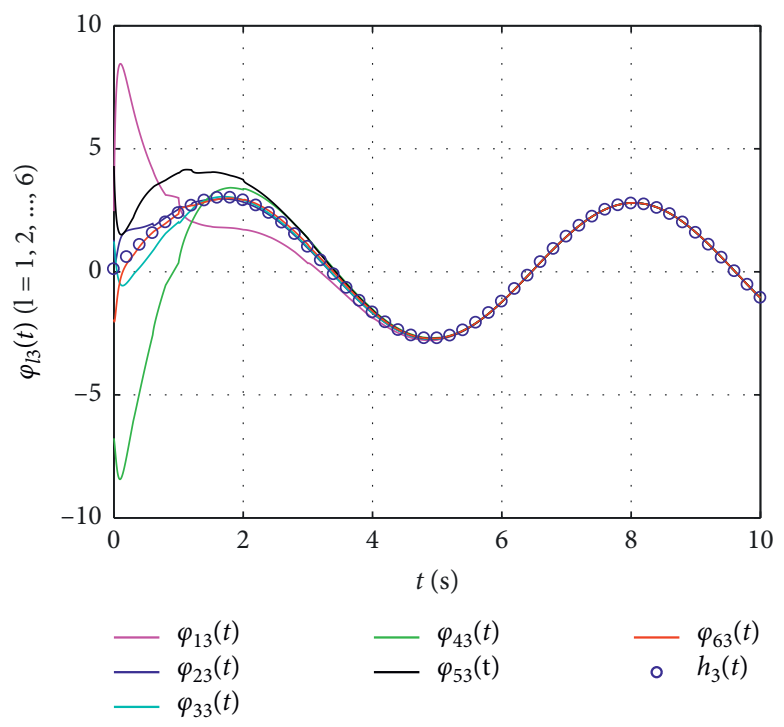

(c)

Figure 2: Trajectories of $\varphi_{l}(l=1,2, \ldots, 6)$. 


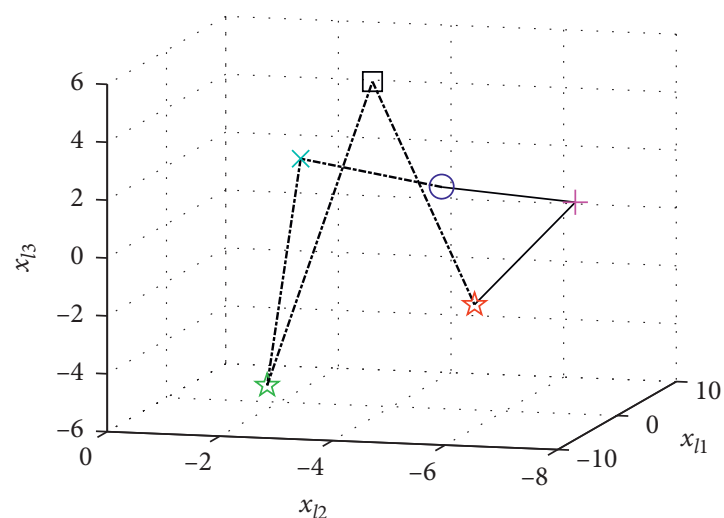

(a)

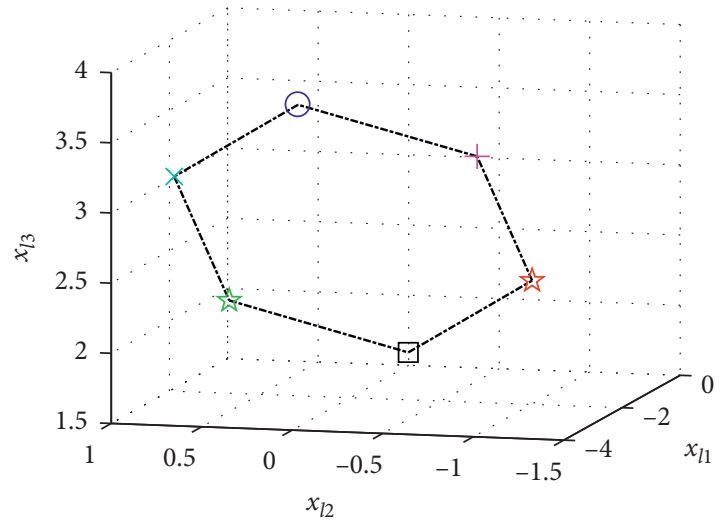

(c)

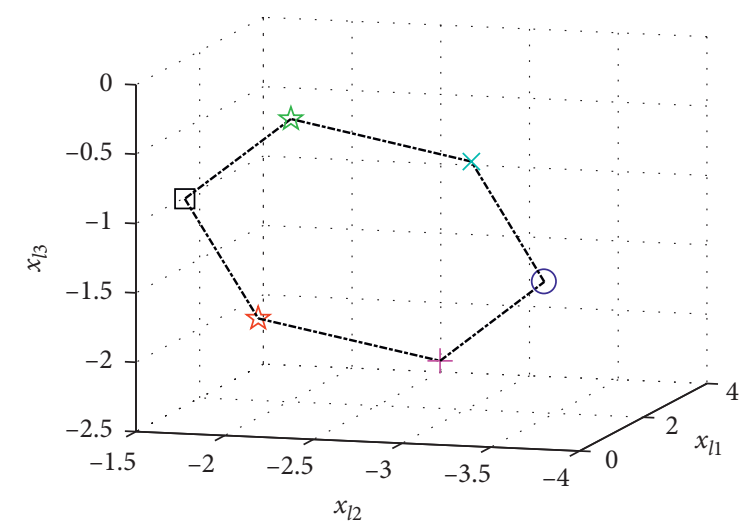

(b)

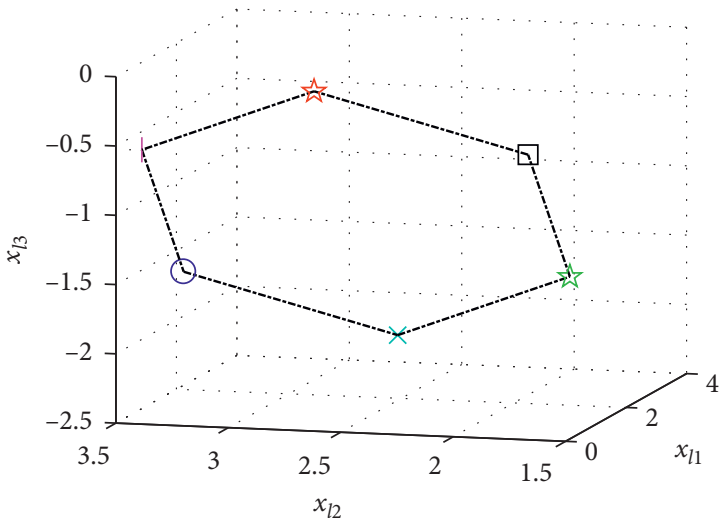

(d)

Figure 3: State snapshots of six agents at different times: (a) $t=0 \mathrm{~s}$; (b) $t=8 \mathrm{~s}$; (c) $t=9 \mathrm{~s}$; (d) $t=10 \mathrm{~s}$.

$$
z_{l}(t)=\left[\begin{array}{c}
\sin \left(t+\frac{(l-1) \pi}{3}\right) \\
\cos \left(t+\frac{(l-1) \pi}{3}\right) \\
-\sin \left(t+\frac{(l-1) \pi}{3}\right)
\end{array}\right], \quad l=1,2, \ldots, 6 .
$$

Based on Theorem 2, the matrix variable and the gain matrix are calculated as

$$
\begin{aligned}
\beta & =8.5573 \\
\widetilde{Q} & =\left[\begin{array}{ccc}
4.1772 & -2.2475 & 4.2758 \\
-2.2475 & 7.3766 & 1.4573 \\
4.2758 & 1.4573 & 20.4770
\end{array}\right], \\
K & =[8.2490,6.9729,-0.6591] .
\end{aligned}
$$

Figure 2 shows the trajectories of $\varphi_{l}(l=1,2, \ldots, 6)$ for the networked agents, where the full curves with different colors denote the $\varphi_{l}(l=1,2, \ldots, 6)$ for the six agents and the sequence of blue circles represents the formation center function. We can see from Figure 2 that the curve of each agent converges to that of the formation center function; that is, they reach the consensus with the states of the formation center functions.

Figure 3 depicts the state snapshots of six agents at $t=0 \mathrm{~s}, t=8 \mathrm{~s}, t=9 \mathrm{~s}$, and $t=10 \mathrm{~s}$, where six agents are depicted by blue circles, pink plusses, red hexagrams, black squares, green hexagrams, and indigo $x$-marks. We can find from Figure 3 that the six agents reach a time-varying regular hexagon around the formation center at different times, which can keep rotating. The above simulation results indicate that network (1) with protocol (2) can reach the leaderless time-varying formation with discontinuous communications.

Example 2. (leader-follower topologies). Consider a leader labeled by 6 and five followers labeled by 1-5, whose dynamics and initial states are the same as the leaderless cases. The switching topologies are given in Figure 4, where the dwell time is set as $0.3 \mathrm{~s}$. The communication time unit and noncommunication time unit are $t \in[s, s+0.8) s$ and $t \in[s+0.8, s+1) s$, respectively, and the corresponding parameters are chosen as the same as the leaderless cases. 

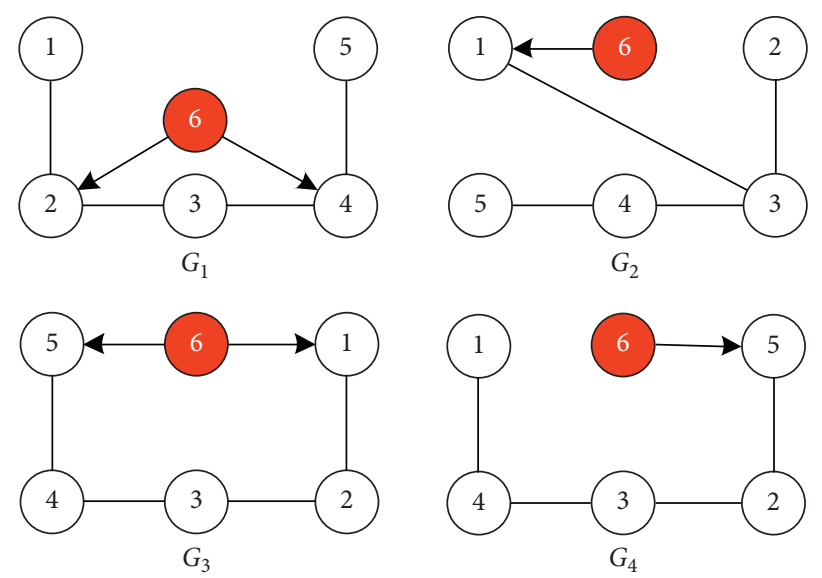

FIGURE 4: Leader-follower switching topologies.

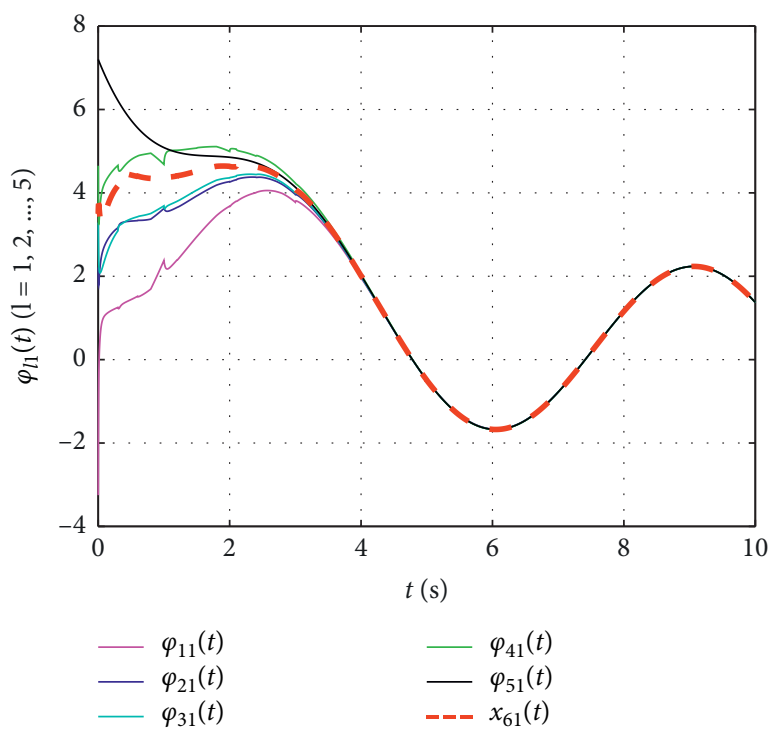

(a)

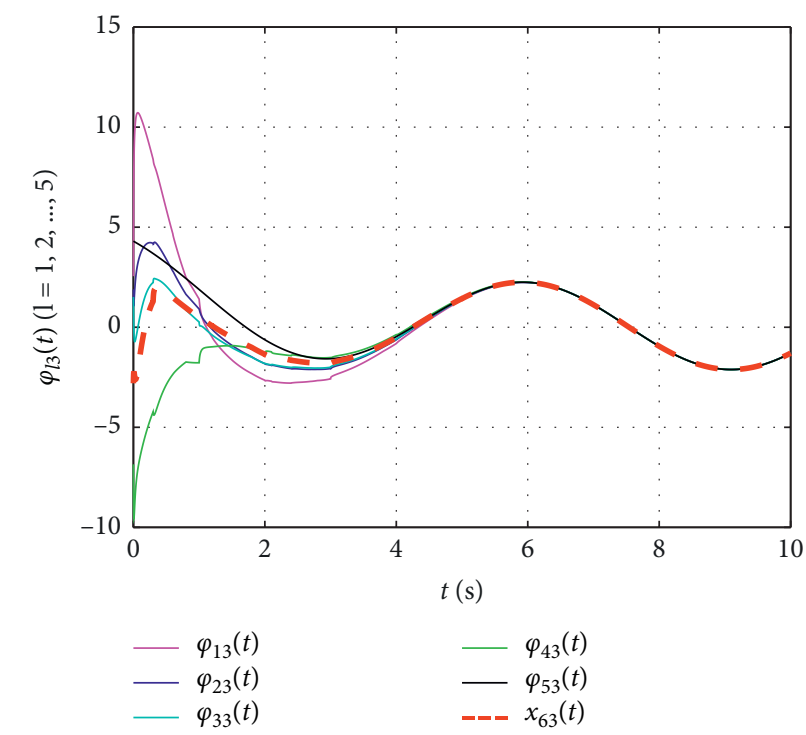

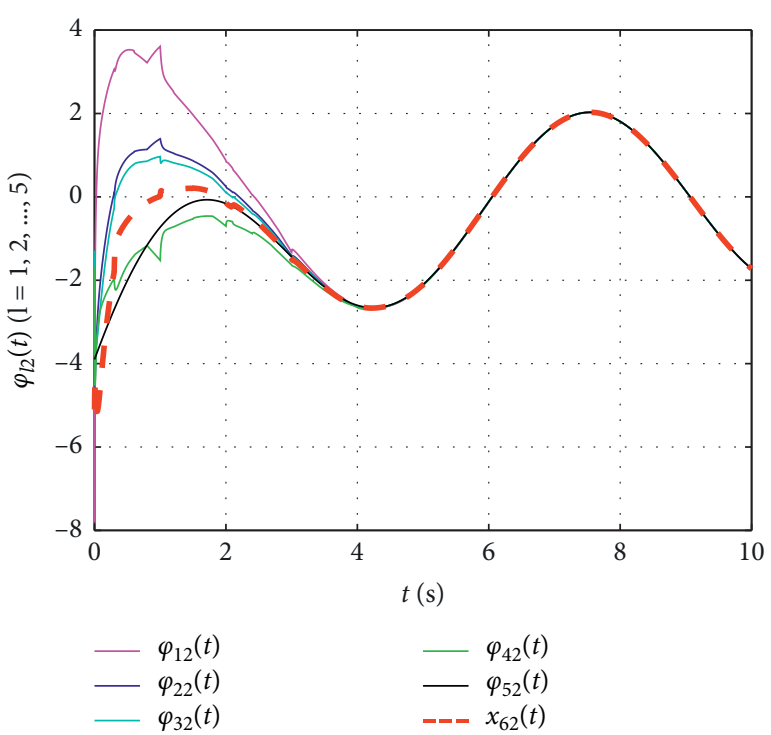

(b)

(c)

FIgURE 5: Trajectories of $\varphi_{l}(l=1,2, \ldots, 5)$ and that of the leader. 


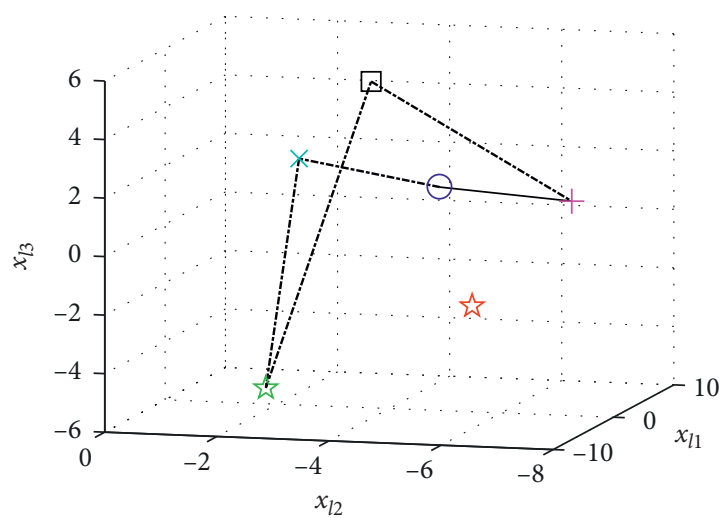

(a)

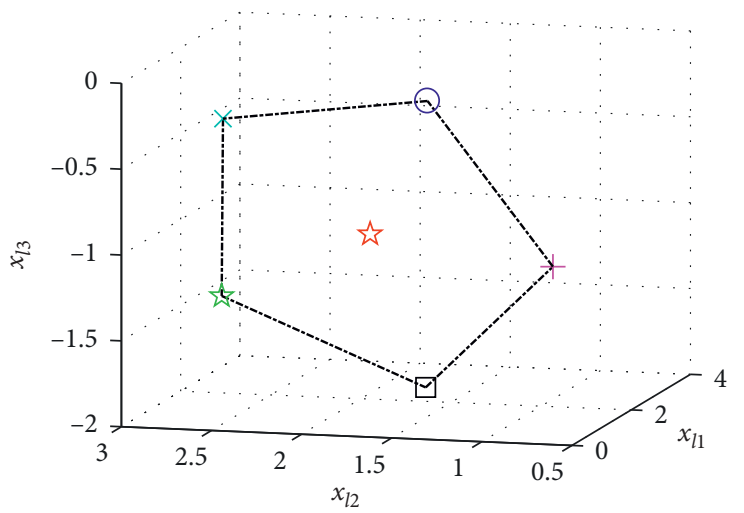

(c)

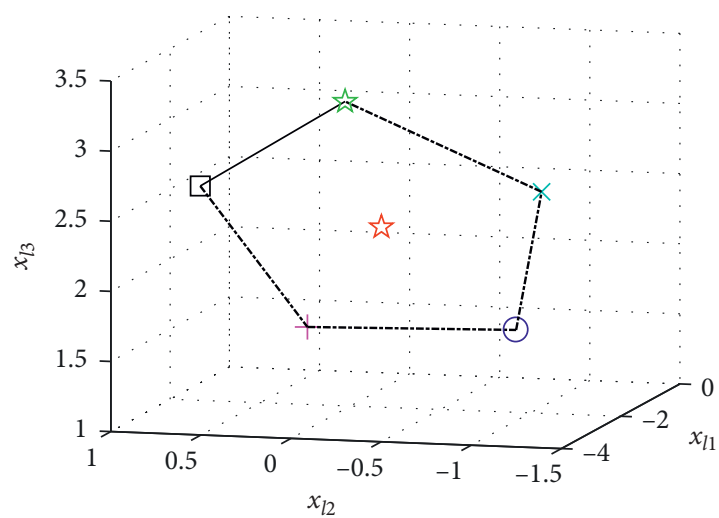

(b)

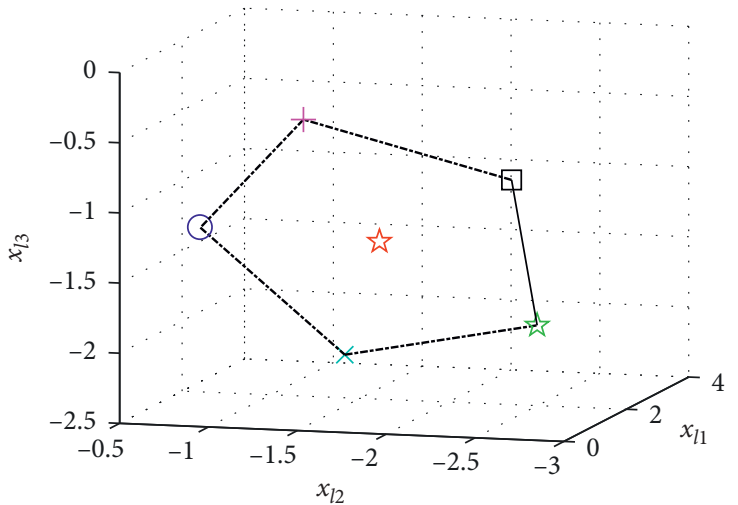

(d)

Figure 6: State snapshots of five followers and the leader at different times: (a) $t=0 \mathrm{~s}$; (b) $t=8 \mathrm{~s}$; (c) $t=9 \mathrm{~s}$; (d) $t=10 \mathrm{~s}$.

The time-varying formation instruction is chosen as

$$
z_{l}(t)=\left[\begin{array}{c}
\sin \left(t+\frac{2(l-1) \pi}{5}\right) \\
\cos \left(t+\frac{2(l-1) \pi}{5}\right) \\
-\sin \left(t+\frac{2(l-1) \pi}{5}\right)
\end{array}\right], \quad l=1,2, \ldots, 5
$$

According to Theorem 3, the matrix variable and the gain matrix are calculated as

$$
\begin{aligned}
\xi & =8.5573, \\
\widetilde{R} & =\left[\begin{array}{ccc}
4.1772 & -2.2475 & 4.2758 \\
-2.2475 & 7.3766 & 1.4573 \\
4.2758 & 1.4573 & 20.4770
\end{array}\right], \\
K_{l f} & =[27.2831,23.0624,-2.1799] .
\end{aligned}
$$

Figure 5 shows the trajectories of $\varphi_{l}(l=1,2, \ldots, 5)$ for five followers and that of the leader, where the full curves with different colors stand for the $\varphi_{l}(l=1,2, \ldots, 5)$ for the five followers and the sequence of red imaginary curves denotes that of the leader. It can be found from Figure 5 that the curves of all followers converge to that of the leader; that is, they can track the trajectory of the leader.

Figure 6 depicts the state snapshots of five followers and the leader at $t=0 \mathrm{~s}, t=8 \mathrm{~s}, t=9 \mathrm{~s}$, and $t=10 \mathrm{~s}$, where they are depicted by blue circles, pink plusses, red hexagrams, black squares, green hexagrams, and indigo $x$-marks. We can find from Figure 3 that the six agents reach a time-varying regular pentagon and keep rotating around the leader, which means that network (26) with protocol (27) can reach the leader-follower time-varying formation with discontinuous communications.

\section{Conclusions}

The leaderless and leader-follower time-varying formation design and analysis for networked agents with discontinuous communications were studied. The leaderless time-varying formation control protocol was proposed via the intermittent control strategy, which contains both the communication time unit and the noncommunication time unit. An explicit formulation of the formation center function was determined, which can describe the formation movement trajectory of the networked agents as a whole. Leaderless time-varying formation design and analysis with discontinuous communications were given, where the formation 
feasibility conditions and discontinuous communication conditions were constructed to ensure the stability of the closed-loop subnetworks. Moreover, the main results of the leaderless cases were extended to the leader-follower cases, where the trajectory of the formation movement was determined by the state of the leader.

\section{Data Availability}

The data used to support this study are included within this article.

\section{Conflicts of Interest}

The authors declare that there are no conflicts of interest.

\section{Acknowledgments}

This work was supported by the National Natural Science Foundation of China (Grant nos. 61867005, 61763040, and 61703411).

\section{References}

[1] R. Olfati-Saber, "Flocking for multi-agent dynamic systems: algorithms and theory," IEEE Transactions on Automatic Control, vol. 51, no. 3, pp. 401-420, 2006.

[2] J. Qu, Z. Ji, C. Lin, and H. Yu, "Fast consensus seeking on networks with antagonistic interactions," Complexity, vol. 2018, Article ID 7831317, 15 pages, 2018.

[3] J. Xi, Z. Fan, H. Liu, and T. Zheng, "Guaranteed-cost consensus for multiagent networks with Lipschitz nonlinear dynamics and switching topologies," International Journal of Robust and Nonlinear Control, vol. 28, no. 7, pp. 2841-2852, 2018.

[4] J. Sun, Z. Geng, Z. Li, and Z. Ding, "Distributed adaptive consensus disturbance rejection for multi-agent systems on directed graphs," IEEE Transactions on Control of Network Systems, vol. 5, no. 1, pp. 202-212, 2018.

[5] J. Xi, C. Wang, X. Yang, and B. Yang, "Limited-budget output consensus for descriptor multiagent systems with energy constraints," IEEE Transactions on Cybernetics, vol. 50, no. 11, pp. 4585-4598, 2020.

[6] Y. Zhang, H. Li, J. Sun, and W. He, "Cooperative adaptive event-triggered control for multiagent systems with actuator failures," IEEE Transactions on Systems, Man, and Cybernetics: Systems, vol. 49, no. 9, pp. 1759-1768, 2019.

[7] L. Consolini, F. Morbidi, D. Prattichizzo, and M. Tosques, "Leader-follower formation control of nonholonomic mobile robots with input constraints," Automatica, vol. 44, no. 5, pp. 1343-1349, 2008.

[8] K.-K. Oh and H.-S. Ahn, "Formation control of mobile agents based on distributed position estimation," IEEE Transactions on Automatic Control, vol. 58, no. 3, pp. 737-742, 2013.

[9] J. Xi, L. Wang, J. Zheng, and X. Yang, "Energy-constraint formation for multiagent systems with switching interaction topologies," IEEE Transactions on Circuits and Systems I: Regular Papers, vol. 67, no. 6, pp. 2442-2454, 2020.

[10] H. Liu, T. Ma, F. L. Lewis, and Y. Wan, "Robust formation control for multiple quadrotors with nonlinearities and disturbances," IEEE Transactions on Cybernetics, vol. 50, no. 4, pp. 1362-1371, 2020.
[11] X. Yang, G. Lin, Y. Liu, F. Nie, and L. Lin, "Fast spectral embedded clustering based on structured graph learning for large-scale hyperspectral image," IEEE Geoscience and Remote Sensing Letters, p. 1, 2020 to be published.

[12] R. Lu, X. Yang, X. Jing et al., "Infrared small target detection based on local hypergraph dissimilarity measure," IEEE Geoscience and Remote Sensing Letters, p. 1, 2020 to be published.

[13] R. Lu, X. Yang, W. Li, J. Fan, D. Li, and X. Jing, "Robust infrared small target detection via multidirectional derivativebased weighted contrast measure," IEEE Geoscience and Remote Sensing Letters, p. 1, 2020 to be published.

[14] N. Cai, M. He, Q. Wu, and M. J. Khan, "On almost controllability of dynamical complex networks with noises," Journal of Systems Science and Complexity, vol. 32, no. 4, pp. 1125-1139, 2019.

[15] Z.-Y. Tan, N. Cai, J. Zhou, and S.-G. Zhang, "On performance of peer review for academic journals: analysis based on distributed parallel system," IEEE Access, vol. 7, pp. 19024-19032, 2019.

[16] W. Ren, "Consensus strategies for cooperative control of vehicle formations," IET Control Theory \& Applications, vol. 1, no. 2, pp. 505-512, 2007.

[17] J. Xi, C. Wang, H. Liu, and L. Wang, "Completely distributed guaranteed-performance consensualization for high-order multiagent systems with switching topologies," IEEE Transactions on Systems, Man, and Cybernetics: Systems, vol. 49, no. 7, pp. 1338-1348, 2019.

[18] L. Wang, J. Xi, Z. Yu, and X. Liu, "Limited-budget finite-time average consensus design for multi-agent systems," IET Control Theory \& Applications, vol. 14, no. 15, pp. 2197-2204, 2020.

[19] J. Xi, M. He, H. Liu, and J. Zheng, "Admissible output consensualization control for singular multi-agent systems with time delays," Journal of the Franklin Institute, vol. 353, no. 16, pp. 4074-4090, 2016.

[20] W. Zou, P. Shi, Z. Xiang, and Y. Shi, "Finite-time consensus of second-order switched nonlinear multi-agent systems," IEEE Transactions on Neural Networks and Learning Systems, vol. 31, no. 5, pp. 1757-1762, 2020.

[21] W. Zou, C. K. Ahn, and Z. Xiang, "Fuzzy-approximationbased distributed fault-tolerant consensus for heterogeneous switched nonlinear multiagent systems," IEEE Transactions on Fuzzy Systems, p. 1, 2020.

[22] W. Zou, P. Shi, Z. Xiang, and Y. Shi, "Consensus tracking control of switched stochastic nonlinear multiagent systems via event-triggered strategy," IEEE Transactions on Neural Networks and Learning Systems, vol. 31, no. 3, pp. 1036-1045, 2020.

[23] J. A. Fax and R. M. Murray, "Information flow and cooperative control of vehicle formations," IEEE Transactions on Automatic Control, vol. 49, no. 9, pp. 1465-1476, 2004.

[24] M. Jafarian, E. Vos, C. De Persis, J. Scherpen, and A. van der Schaft, "Disturbance rejection in formation keeping control of nonholonomic wheeled robots," International Journal of Robust and Nonlinear Control, vol. 26, no. 15, pp. 3344-3362, 2016.

[25] H. Du, G. Wen, Y. Cheng, Y. He, and R. Jia, "Distributed finite-time cooperative control of multiple high-order nonholonomic mobile robots," IEEE Transactions on Neural Networks and Learning Systems, vol. 28, no. 12, pp. 29983006, 2017.

[26] L. Brinon-Arranz, A. Seuret, and C. Canudas-de-Wit, "Cooperative control design for time-varying formations of multi- 
agent systems," IEEE Transactions on Automatic Control, vol. 59, no. 8, pp. 2283-2288, 2014.

[27] R. Rahimi, F. Abdollahi, and K. Naqshi, “Time-varying formation control of a collaborative heterogeneous multi agent system," Robotics and Autonomous Systems, vol. 62, no. 12, pp. 1799-1805, 2014.

[28] X. Dong, Y. Zhou, Z. Ren, and Y. S. Zhong, “Time-varying formation tracking for second-order multi-agent systems subjected to switching topologies with application to quadrotor formation flying," IEEE Transactions on Industrial Electronics, vol. 64, no. 6, pp. 5014-5024, 2016.

[29] L. Wang, J. Xi, M. He, and G. Liu, "Robust time-varying formation design for multiagent systems with disturbances: extended-state-observer method," International Journal of Robust and Nonlinear Control, vol. 30, no. 7, pp. 2796-2808, 2020.

[30] X. Dong and G. Hu, "Time-varying formation tracking for linear multiagent systems with multiple leaders," IEEE Transactions on Automatic Control, vol. 62, no. 7, pp. 36583664, 2017.

[31] J. Shao, W. X. Zheng, T.-Z. Huang, and A. N. Bishop, "On leader-follower consensus with switching topologies: an analysis inspired by pigeon hierarchies," IEEE Transactions on Automatic Control, vol. 63, no. 10, pp. 3588-3593, 2018.

[32] R. Wang, "Adaptive output-feedback time-varying formation tracking control for multi-agent systems with switching directed networks," Journal of the Franklin Institute, vol. 357, no. 1, pp. 551-568, 2020.

[33] L. Wang, J. Xi, B. Hou, and G. Liu, "Limited-budget consensus design and analysis for multiagent systems with switching topologies and intermittent communications," IEEE/CAA Journal of Automatica Sinica, vol. 8, 2021.

[34] J. Sun and Z. Wang, "Consensus of multi-agent systems with intermittent communications via sampling time unit approach," Neurocomputing, vol. 397, pp. 149-159, 2020.

[35] W. Qin, Z. Liu, and Z. Chen, "A novel observer-based formation for nonlinear multi-agent systems with time delay and intermittent communication," Nonlinear Dynamics, vol. 79, no. 3, pp. 1651-1664, 2015.

[36] C. Godsil and G. Royal, Algebraic Graph Theory, Springer, New York, NY, USA, 2001. 